\title{
Calidad educativa y educación ambiental en universidades de Huancayo, Perú
}

\author{
Educational quality and environmental education \\ in universities of Huancayo, Perú
}

\author{
Eusebio Zenón Castro León ${ }^{1,2}$ \\ ${ }^{1}$ Universidad Continental, ${ }^{2}$ Universidad Nacional del Centro del Perú
}

\section{RESUMEN}

El objetivo fue evaluar la calidad educativa y contrastar con la educación ambiental en la Universidad Nacional del Centro del Perú (UNCP) y la Universidad Peruana Los Andes (UPLA) de Huancayo. El tipo de investigación fue aplicada, nivel descriptivo, diseño no experimental, transversal, y correlacional. La muestra aplicada en la UNCP fue de 1052, con un auto-ponderado de 1020, y en la UPLA 950, con un auto-ponderado de 951. Respecto a las técnicas para la recolección de datos en ambas variables fue la encuesta, y como instrumento se empleó el cuestionario, el mismo que fue sometida a la prueba de confiabilidad Alfa de Cronbach. Los resultados referente a calidad educativa en cada lineamiento se observa que efectivamente los recursos educacionales e infraestructura presenta el mayor índice de discrepancias y que el lineamiento de contribución intelectual, imagen e internacionalización es el que posee menor índice de discrepancias; con relación a educación ambiental se nota que el porcentaje de discrepancia es mayor que el porcentaje de opinión favorable; en relación a la variable calidad educativa y educación ambiental tienen el mayor número de relaciones encontradas. En conclusión, ambas universidades no estarían brindando calidad educativa, asimismo, no existe un compromiso con la educación ambiental y sobre todo con la protección del medio ambiente, demostrándose que ambas variables estudiadas se relacionan recíprocamente, lo cual por lo menos permite suponer que existe un factor común que los induce a ello.

Palabras clave: Calidad educativa, educación ambiental.

\section{ABSTRACT}

The objective was to evaluate the educational quality and contrast with environmental education in the Universidad Nacional del Centro del Perú (UNCP) and the Universidad Peruana Los Andes (UPLA) in Huancayo. The type of research was applied, descriptive level, non-experimental, transversal, and correlational design. The sample applied in the UNCP was 1052, with a self-weighting of 1020, and in the UPLA 950, with a self-weighting of 951. Regarding the techniques for data collection in both variables survey was the survey, and as instrument was used the questionnaire, which was subjected to the Cronbach Alpha reliability test. The results regarding educational quality in each guideline show that educational resources and infrastructure have the highest index of discrepancies and that the guideline of intellectual contribution, image and internationalization is the one with the lowest index of discrepancies; in relation to environmental education, the percentage of discrepancy is higher than the favorable opinion percentage; in relation to the variable educational quality and environmental education have the highest number of relationships found. In conclusion, both universities would not be providing educational quality; in addition, there is no commitment to environmental education and above all to the protection of the environment, demonstrating that both variables are related to each other, which at least suggests that there is a common factor that induces them.

Keywords: Quality education, environmental education.

Historial del artículo:

Recibido, 17 de febrero 2016; aceptado, 16 de mayo de 2016; disponible en línea, 20 de julio de 2016

* Docente en la Universidad Nacional del Centro del Perú, Universidad Continental en Huancayo, Perú.

Correo: zenoncastroleon3@hotmail.com 


\section{INTRODUCCIÓN}

El desarrollo de la educación superior en América Latina se ha caracterizado durante las últimas décadas por una creciente demanda de acceso y resultados, en un contexto de inestabilidad económica e importantes transformaciones político-sociales. Esto ha provocado en la región el aumento de estudiantes, expansión en tamaño y número de universidades, originando con ello un mercado caracterizado por la heterogeneidad de la oferta y diversidad estudiantil.

Los gobiernos han reconocido que ha existido una creciente preocupación por enfrentar adecuadamente este fenómeno $y$, en particular, hay un esfuerzo importante de las universidades por mejorar la calidad educativa, incluyendo la eficiencia y eficacia de la función docente. La función docente debe entenderse como una labor orientada a la formación y desarrollo de las personas, portadora de una acreditación social válida dentro de la cultura en la cual se realiza. Es decir, se trata de una actividad explícitamente certificada que redunda en la transformación de personas y en la preparación de profesionales necesarios para una sociedad.

El sistema educativo considera las instituciones superiores como centrales para la formación de profesionales de las distintas actividades que necesita para su desarrollo. Se establece que la educación superior es una condición para la futura prosperidad, convirtiéndose en un pilar para la competitividad económica y movilidad social.

No obstante, para que las nuevas generaciones puedan enfrentar las demandas sociales de manera autónoma y eficaz, se requiere que su proceso educativo sea guiado por docentes competentes en la enseñanza de conocimientos teórico-prácticos pertinentes para desenvolverse en el mundo actual (UNESCO, 1997).

Asimismo, es deseable lograr una alta calidad en la educación procurando que esa educación alcance la mayor equidad y pertinencia social. Es decir, esa educación sea más pertinente a las necesidades de los individuos y de los grupos sociales a los que pertenecen. Por ello hoy en día la calidad educativa es un aspecto importante en toda institución superior, caso específico de la Universidad.

Esta preocupación no es ajena a las universidades peruanas, mucho menos a las universidades de Huancayo. Al igual que en otras esferas educativas, en la ciudad de Huancayo se tiene a la Universidad Nacional del Centro del Perú, Universidad Peruana Los Andes, y Universidad Continental (estas dos últimas de gestión privada) y algunas filiales de universidades de otros lugares del país (Alas Peruanas, Los Ángeles de Chimbote, César Vallejo).
Sin embargo, no basta tener una calidad educativa, por decir excelente, si no también es necesario que vaya acompañada por una buena educación ambiental hacia la población estudiantil e incluso los docentes. Pues sin educación ambiental no hay desarrollo sostenible y si no existe desarrollo sostenible no existe progreso. Nuestra realidad nos indica que la universidad no va acorde con los requerimientos de la sociedad actual ya que no es participe en la solución de sus problemas como es el caso del cambio climático, protección de la naturaleza y la biodiversidad, los cuales son elementos claves para el desarrollo humano. Por ello la propuesta de incluir en el presente trabajo de investigación lo referente a Educación Ambiental lo cual no implica solo un cambio de paradigma epistemológico, ético y estratégico sino que representa una forma progresista de modernidad que propone la preservación de sus valores, prácticas y privilegia a la racionalidad instrumental mediante el saber científico y tecnológico.

Se considera que la Educación Ambiental permite entender, interpretar y actuar de manera adecuada frente a la problemática medioambiental, pues ella constituye el eslabón clave para los cambios en las actitudes y valores de los docentes, estudiantes con respecto al medio. La educación ambiental es el eje fundamental para impulsar los procesos de prevención del deterioro ambiental. La toma de conciencia de la ciudadanía en este proceso es crucial e implica una educación que fomente valores y hábitos para lograr un medio ambiente en equilibrio y sostenible.

La Educación Ambiental es la estrategia más adecuada para superar la crisis ambiental global del fin del milenio (García, 1997; Junyent, 1997), reorientando las normas de comportamiento, los métodos de pensamiento, inculcando una nueva moral (Pardo, 1995; TECA, 1991) y nuevos estilos de vida (COLOM, 1988). Esto será posible, si se cumplen los objetivos de la Educación Ambiental, la cual debe modificar los hábitos, las actitudes, valoraciones (Catalán, 1993), cambios en el consumo y en los modelos de producción (UNESCO, 1997), permitiendo la consecución a nivel local, nacional, regional y mundial de un tipo de desarrollo que garantice las condiciones de vida de los presentes y futuras generaciones dando lugar así a un desarrollo sustentable (López, 1966; Oliva, 1997; González, 1995). Este desarrollo de sustentabilidad no deberá abarcar solamente el medio ambiente, sino también a la pobreza, la población, la salud, la seguridad alimentaria, la democracia, los derechos humanos y la paz (UNESCO, 1997).

Hoy en día, la educación ambiental no funciona si no se convierte en tangible y la podemos aplicar en nuestra vida diaria, sin que podamos ver resultados reales que tengan un efecto de cambio en nuestro entorno inmediato. Es ello, lo que impulsa el presente 
trabajo de investigación realizado en la ciudad de Huancayo, en la Universidad Nacional del Centro (UNCP) y la Universidad Peruana Los Andes (UPLA). Se pretende buscar una relación entre la calidad educativa y la educación ambiental, por ello el diseño de investigación corresponde a una Investigación descriptiva tipo transversal - comparativa a través de encuestas realizadas tanto a docentes, estudiantes y a administrativos de ambas universidades, pues la finalidad es obtener un consolidado de los principales actores que forman parte del quehacer universitario.

Por ello esperamos que los resultados obtenidos sirvan de base y de cimiento a las principales autoridades de ambas universidades en estudio y del resto de universidades del país; y, a partir de ello proponer alternativas de solución a las deficiencias encontradas.

El problema principal planteado fue: $\grave{2}$ En qué medida la calidad educativa tiene relación con la educación ambiental en las universidades de Huancayo? Se evaluó la calidad educativa de la Universidad Nacional del Centro del Perú y de la Universidad Peruana Los Andes y contrastó con la educación ambiental, lo cual permitió visualizar la relación entre la calidad educativa con la educación ambiental.

Existen pocos trabajos, referidos al tema que se ha realizado en nuestro país, de ahí la importancia del presente trabajo de investigación en el que se abordará a la evaluación de la calidad educativa y educación ambiental de dos universidades, nacional y privada y determinar la relación entre la calidad educativa y la educación ambiental en ambas universidades.

La hipótesis definida fue que, la calidad educativa tiene relación directa con la educación ambiental, en la Universidad Nacional del Centro del Perú y Universidad Peruana Los Andes.

\section{MATERIAL Y MÉTODOS}

El tipo de investigación fue aplicada, nivel descriptivo, diseño no experimental, transversal, descriptivo y correlacional.

La población considerada en la investigación fue las universidades en estudio y en la que se hallan comprendidos docentes, estudiantes y administrativos, definida de la siguiente manera:

- Universidad Nacional del Centro del Perú: Docentes nombrados (616), estudiantes (9 723), administrativos nombrados (321), total (10 660). $\mathrm{Se}$ exceptuaron a los docentes contratados y administrativos contratados. La muestra calculada corresponde a una muestra probabilística de 1052 , con una muestra auto-ponderada de 1020.
- Universidad Peruana Los Andes: Docentes nombrados (99), estudiantes (8 886), Administrativos nombrados (200), total (9 185). Se exceptuaron a los docentes contratados y administrativos contratados. La muestra calculada corresponde a una muestra probabilística de 950, con una muestra autoponderada de 951.

Respecto a las técnicas para la recolección de datos para la variable calidad educativa fue la encuesta, y como instrumento se empleó un cuestionario, propuesto por el consorcio de universidades y dentro de ellas nuestros indicadores.

Para la variable Educación Ambiental también se utilizó una encuesta basado en un cuestionario el mismo que fue sometida a la Prueba de confiabilidad Alfa de Crombach.

Para el análisis de datos, se utilizó la estadística descriptiva (tablas, diagramas de pareto y gráfico de líneas o perfiles), la estadística inferencial (prueba $\mathrm{X}^{2}$ para independencia de criterios y el análisis de varianza para comparación de opiniones de grupos o estratos).

Para el análisis de la relación desempeño docente y calidad educativa se empleó la prueba de $X^{2}$ para Independencia de criterios (para ver si existe o no relación entre variables).

Las variables calidad ambiental y educación ambiental, utilizaron cada uno, independientemente la prueba de Kruskal - Wallis, para determinar la discrepancia entre los Ítems de cada lineamiento; el análisis univariado y estadística descriptiva.

Para la relación de ambas variables se utilizó la prueba de independencia de criterios de $X^{2}$, coeficiente de contingencia.

\section{RESULTADOS}

\section{Calidad Educativa}

Respecto al lineamiento "proyecto institucional" la prueba de Kruskal-Wallis, se reporta 5 items con discrepancias significativas de un total de 10, lo que indica que en este rubro existe $50 \%$ de discrepancias de opinión de los encuestados y esto se da para ambas universidades. Estos items significativos corresponden a los niveles de autoridad, niveles de dependencia, niveles de coordinación y a los procedimientos dentro de la institución. Involucra que un $50 \%$ de los encuestados desconocen los niveles de autoridad, niveles de dependencia y/o niveles de coordinación lo que no permite un adecuado funcionamiento administrativo de ambas universidades. 
Referente al lineamiento "programa académico" el resumen de la prueba de Kruskal -Wallis, muestra 4 ítems significativos de un total de 9, esto representa un $48 \%$ de discrepancias de opinión en los encuestados (docentes, estudiantesy administrativos). De estos cuatro ítems significativos llama la atención las opiniones referentes al plan curricular de ambas universidades, donde no existe consenso en determinarlo si es o no integral, y estas diferencias de opinión tienen una discrepancia de hasta el $99 \%$. El $48 \%$ de los encuestados opinan que los planes curriculares de ambas universidades no son los más adecuados, no son integrales ni humanistas, estos resultados obtenidos contradicen lo propuesto como visión de cada una de las universidades en estudio, así la UNCP tiene como visión ser una "Universidad humanista e innovadora, generadora de ciencia y tecnología, líder en el desarrollo sostenible" y la visión de la UPLA "Seremos una universidad científica, tecnológica, humanista, líder y competitiva, brindando servicios académicos de de los mismos. En el caso de la UNCP el 80,52 \% de los docentes son nombrados, a tiempo completo y en promedio tienen a su cargo 3 asignaturas como máximo. Mientras que en la UPLA el 9,82 \% de docentes son nombrados, 39,62 \% se encuentran a tiempo completo, el $60,38 \%$ a tiempo parcial y a cada uno de ellos cuenta con 4 asignaturas a su cargo como mínimo. Estos resultados contravienen lo recomendado por la practica pedagógica e incluso su propia normatividad, así la UNCP emitió la Resolución № 00170-CU-2005 de fecha 11 de mayo del 2005, donde determinan que para la realización del cálculo de la Estructura Académico Organizacional se considera que por cada 10 estudiantes debe haber un docente y que cada docente debe estar a cargo de dos asignaturas, lo que en la práctica no se cumple en ninguna de las dos universidades.

Respecto al lineamiento "estudiantes y servicios estudiantiles" el resumen de la prueba de Kruskal -

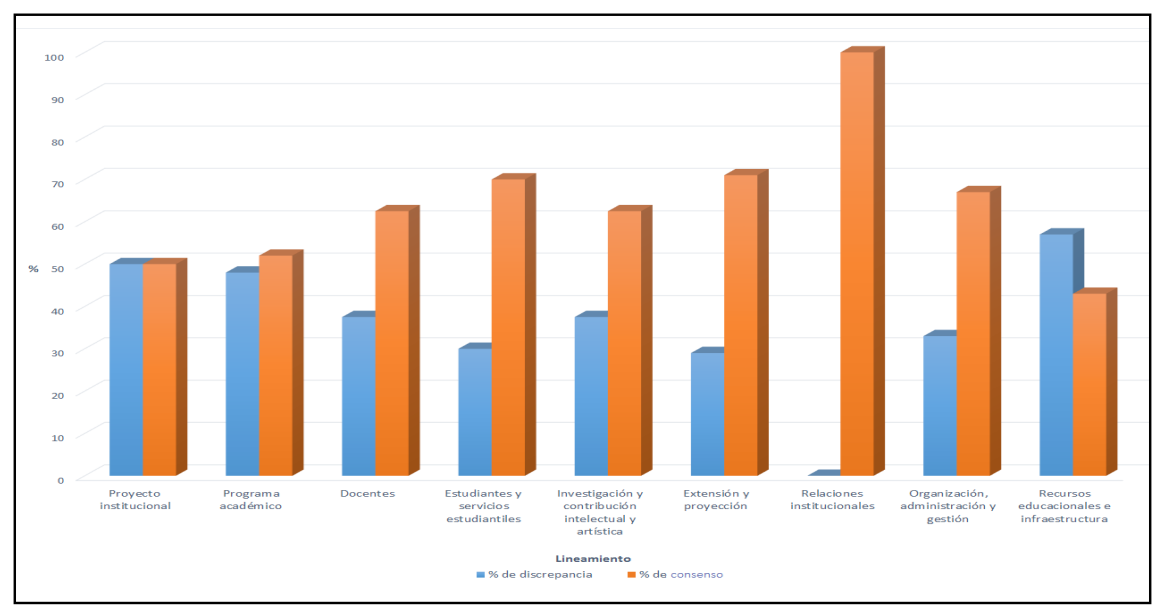

Figura 1: Índice discrepancia, variable Calidad Educativa.

calidad, realizando innovación científica - tecnológica y gestión empresarial, consolidando una institución involucrada en el desarrollo regional y nacional" lo que conlleva a replantear la visión y misión de ambas universidades, asimismo se deberá de actualizar sus planes curriculares acorde a las necesidades de la sociedad y al avance científico.

Referente al lineamiento "docentes", el resumen de la prueba de Kruskal-Wallis, se reporta hasta 3 ítems significativos de un total de 8 , lo que significa que existe un porcentaje de discrepancia del $37,5 \%$ o sea una igualdad de opinión del 62,5\%.

Con respecto a la relación entre el número de docentes y el número de estudiantes, en el caso de la UNCP esta relación, en promedio, es de 12,71. De los tres ítems significativos se consideran como los más importantes y corresponden al número adecuado de docentes que debe tener la facultad y a la dedicación laboral
Wallis, reporta tres ítems significativos de un total de 10 , esto significa que existe una discrepancia del 30 $\%$. De estos ítems significativos se considera que el más importante es que la unidad académica se preocupa por incentivar la asistencia estudiantil a las actividades curriculares y que se debe brindar todo el apoyo del servicio a los estudiantes en cuanto a actividades académicas.

Referente al lineamiento "contribución intelectual y artística", la Constitución Política del Perú en su Artículo $N^{\circ} 18$ a la letra dice "La educación universitaria tiene como fines la formación profesional, la difusión cultural, la creación intelectual y artística y la investigación científica y tecnológica". Asimismo en una encuesta realizada a los docentes de la UNCP en mayo del 2005, demuestran que el $54,3 \%$ de los docentes manifiestan que, la política de investigación actual tiene un nivel de aceptación de muy poco o poco, para contribuir al logro de la misión y visión institucional; igualmente el 
$55,6 \%$ de ellos manifiestan que los programas y líneas actuales tampoco sirven a este propósito, condiciones que sugieren que la política de investigación adoptada por el Centro de Investigación y la Organización de programas y líneas, no están definidas en relación con las necesidades institucionales. Por otro lado, el $63,5 \%$ de los encuestados manifiestan que muy poco $\circ$ poco de los resultados de investigación son incorporados en los procesos de enseñanza, y el $73,4 \%$ de ellos afirman que muy poco o poco de dichas investigaciones contribuyen a la innovación y al desarrollo científico tecnológico. Ambos resultados corroboran las deficiencias que se hallan vigentes en las universidades en estudio. De la investigación realizada el resumen de la prueba de Kruskal - Wallis reporta hasta tres ítems significativos de un total de 8, esto representa el 37,5\% de discrepancias en nuestros informantes. De estos tres ítems significativos llama la atención el referido a que la universidad promueve la realización de actividades culturales y artísticas, estas se realizan en muy pocas oportunidades al año en ambas universidades. Estos resultados indican claramente que las investigaciones que se realizan en ambas universidades no se orientan a generar conocimiento para reforzar la formación académica de los estudiantes, tampoco tienen interés para un proceso de desarrollo científico realista de la región central del país.

Referente al lineamiento extensión y proyección social, la prueba Kruskal - Wallis indica 2 ítems significativos de un total de 7 , esto representa un $29 \%$ de discrepancias; estos ítems significativos se refieren a la participación de los docentes, estudiantes y personal administrativo en actividades de extensión y proyección social. Se puede interpretar, de los resultados obtenidos de ambas universidades, que la proyección social en el caso de la UNCP es dispersa y de poca transcendencia, mientras que la UPLA no cumple con esta función.

Referente al lineamiento "relaciones institucionales, imagen, comunicación e internacionalización", el resumen de la prueba de Kruskal - Wallis no reporta ninguna discrepancia.

Referente al lineamiento "organización, administración y gestión", el reporte de la prueba de Kruskal-Wallis indica 2 ítems significativos de un total de 6, es decir que solo existe un $33 \%$ de discrepancias. De estos 2 ítems significativos llama la atención la eficiencia del personal administrativo, este punto tiene serias discrepancias, según la opinión de los encuestados, el personal administrativo es poco eficiente en las labores que desempeñan en ambas universidades.

Referente al lineamiento "recursos educacionales e infraestructura", el reporte de la prueba de Kruskal Wallis de 14 ítems evaluativos 8 resultaron significativos, lo que significa que existe un $57 \%$ de discrepancia en los encuestados, estos ítems significativos se refieren a las vías de acceso a la universidad, del servicio médico, al cuidado de las vitrinas entre otros aspectos de carácter administrativo, asimismo observan los encuestados que se deben efectuar los mantenimientos de las áreas verdes, y del entorno de cada universidad.

La variable calidad educativa muestra que el lineamiento recursos educacionales e infraestructura presenta el mayor índice de discrepancias y que el lineamiento de contribución intelectual, imagen e internacionalización es el que posee menor índice de discrepancias.

\section{Educación ambiental}

Respecto a conocimientos ambientales, el modelo ANOVA-I, muestra que no existe diferencias significativas de opinión en el grupo de encuestados, es decir en los docentes, estudiantes de la UNCP y de la UPLA; esto significa que los encuestados se encuentran al mismo nivel. Sin embargo, lo resaltante de este hecho radica que en ninguno de nuestros cuatro grupos de encuestados "aprobó" el examen de conocimientos, así los docentes de la UNCP tuvieron un promedio de 8,10; los docentes de la UPLA tuvieron un promedio de 8,40 ; los estudiantes de la UNCP un promedio de 8,95 y los estudiantes de la UPLA 8,76; también se observa un alto coeficiente de variabilidad en los cuatro grupos de encuestados, lo que indica que hubieron notas extremas. Similar resultado obtuvo María Mila en un estudio realizado en la Universidad Autónoma de Barcelona y dice "los resultados obtenidos, respecto a educación ambiental nos demuestran que éste es un tema complejo, poco estudiado y escasamente atendido en los planes y programas educativos (Mila, 2000)".

Es necesario destacar que son las instituciones públicas y privadas las llamadas a promover, coordinar acciones a favor de la educación ambiental y conservación del medio ambiente, junto con el aporte de movimientos ecológicos (Giménez, 1992). Recordar también que lo ambiental es un componente de la cultura al igual que la educación y que como está sólo puede ser aprehensible si es corroborado con aporte de un sistema cultural múltiple y multifuncional (Gonzalo, 1996).

Respecto a valores ambientales, el resumen de la prueba de Kruskal -Wallis reporta doce ítems significativos de un total de 14 , lo que indica que existe un $85,7 \%$ de discrepancia altamente significativa, este resultado muestra que "carecemos "de valores ambientales, o si lo tenemos estos valores son muy limitados.

Referente a actitudes ambientales, la prueba de Kruskal -Wallis, reporta 12 ítems significativos de un total de 18; esto representa el $67 \%$ de discrepancias 


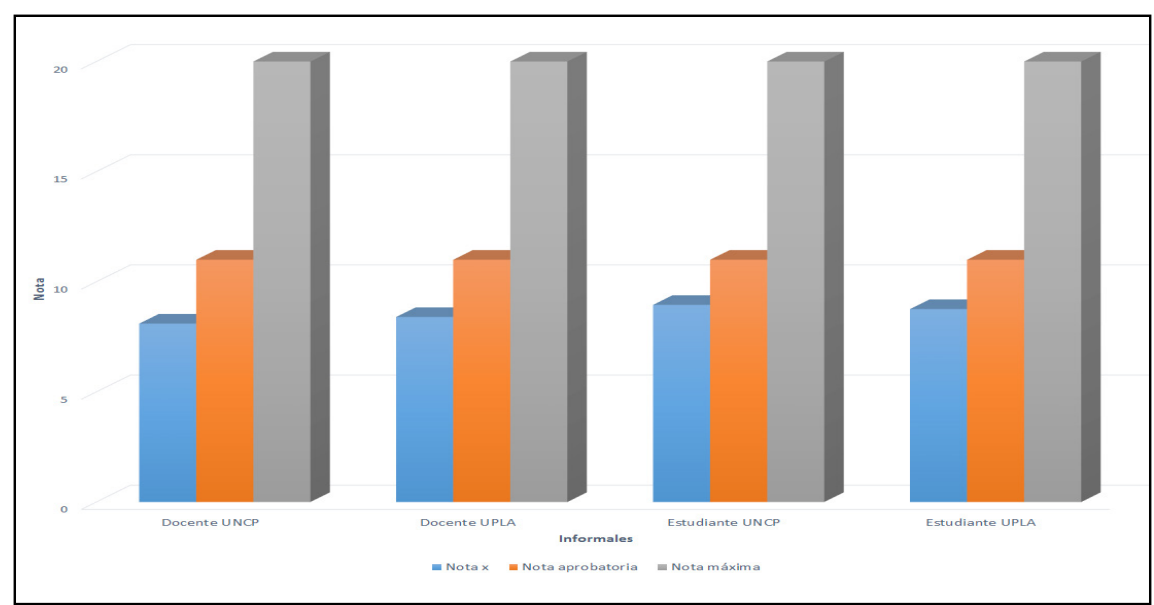

Figura 2: Índice discrepancia, variable Educación Ambiental, sub variable conocimientos.

en los encuestados, por ejemplo actitudes tales como el ahorro de energía, el ahorro de agua, no se cultivan ni se practican.

Referente al rubro de habilidades y competencias, el reporte de la prueba de Kruskal-Wallis, de 12 ítems evaluativos, 10 resultaron con discrepancias significativas, esto indica que el $83 \%$ de los encuestados tienen opiniones diferentes, y tan solo un $16 \%$ poseen opiniones concordantes; esta situación nos lleva a pensar que existen falencias en lo referente a habilidades y /o competencias para afrontar cambios inesperados. Asimismo del análisis que se puede realizar es que las personas no están habituadas a reciclar las cosas ni mucho menos a rehusar.

En lo referente a la variable calidad educativa se encuentran aprobados aunque con un puntaje mínimo; no es lo mismo el resultado obtenido en educación ambiental, donde se encuentran desaprobados.

\section{Relación calidad educativa y educación ambiental}

El análisis de relaciones entre calidad educativa y educación ambiental tanto en la Universidad Nacional de Centro del Perú como la Universidad Privada Los Andes, reporta hasta 260 relaciones significativas, de éstas 75 corresponden al lineamiento recursos educacionales e infraestructura vs. educación ambiental, representando un $29 \%$ del total de las relaciones, lo que significa que el rubro infraestructura y los recursos para la educación tienen relación directa con la educación ambiental, por ello es necesario dotar de más presupuesto a las Universidades para ejercer una mejor labor de enseñanza y específicamente mejorar nuestra educación ambiental.

El segundo lineamiento en importancia es el proyecto institucional y lineamiento investigación y contribución intelectual que tienen 34 relaciones con la educación ambiental, esto significa el $13 \%$ del total de las relaciones.

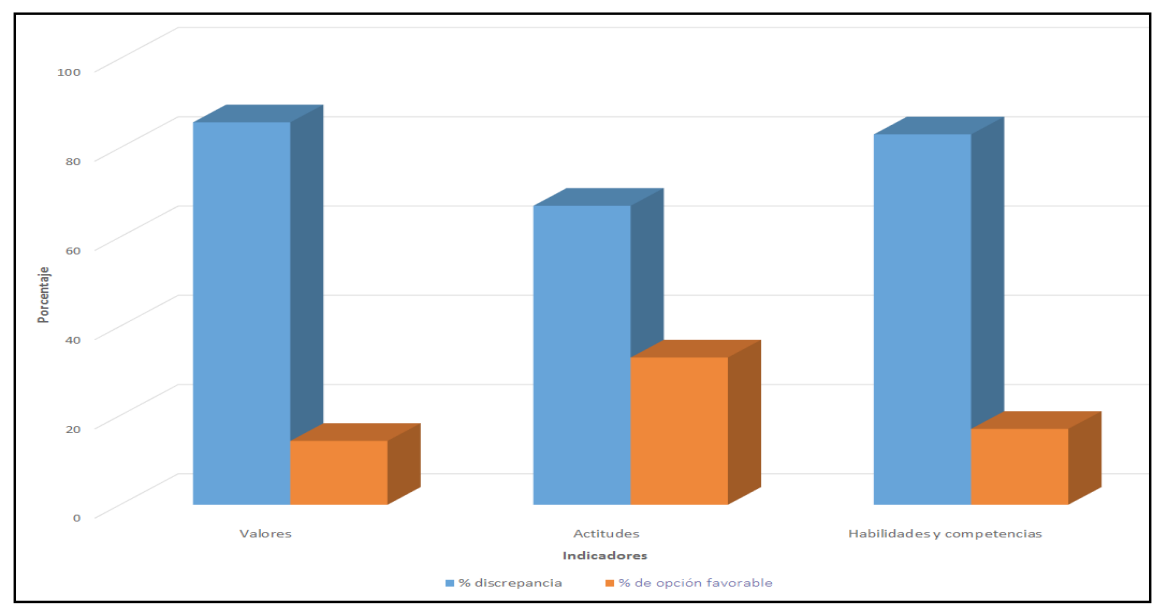

Figura 3: Índice discrepancia, variable Educación Ambiental, otras sub variables. 


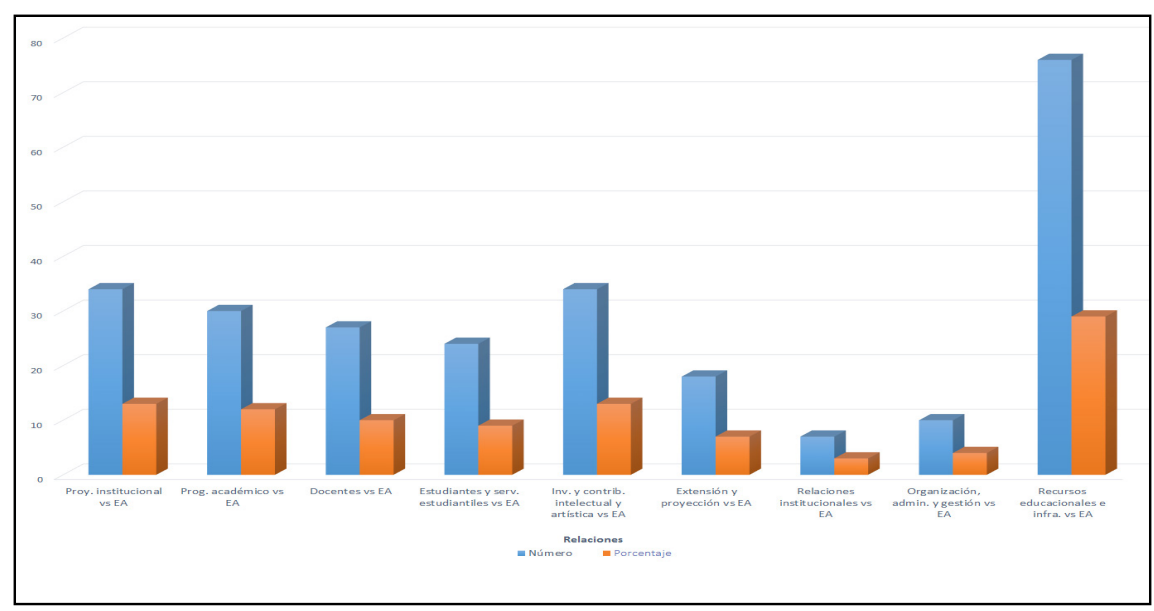

Figura 4: Relación Calidad Educativa (CE) y Educación Ambiental (EA).

En cuanto al lineamiento proyecto institucional se debe establecer y/o difundir adecuadamente los objetivos, misión y visión de cada Universidad; y, que la educación ambiental también debería ser un objetivo importante dentro de la educación universitaria, lo que significaría que la educación ambiental se debería impartir en todas las carreras, en una forma transversal.

De igual forma se encontró que el lineamiento investigación y contribución intelectual también guardan relación con la educación ambiental, significa que las universidades deben invertir en investigar aspectos ambientales si es posible en todas las facultades y en ellas hacer participar a los estudiantes según lo establecen los parámetros de acreditación de universidades.

En el tercer lugar de importancia está el lineamiento docente que posee 28 relaciones de 260 , significando el 10,7\%, es decir que también los docentes tienen relación directa con la educación ambiental, en otras palabras los docentes tienen la gran responsabilidad de enseñar, conducir y dar ejemplo a los estudiantes en cuanto a educación ambiental, pero para ello es necesario que se capaciten en aspectos ambientales. En cuarto lugar, de importancia esta el lineamiento estudiantes y servicios estudiantiles con 24 relaciones encontradas de un total de 260 , equivalente a un 9,2 $\%$, lo que nos manifiesta que también los estudiantes tienen mucho que ver con la Educación Ambiental pues hoy y en el futuro pasarán a ser actores principales en la conservación del ambiente.

El porcentaje de relaciones encontradas de todos los lineamientos de la variable calidad educativa y la variable educación ambiental, se aprecia que efectivamente el lineamiento de recursos educacionales e infraestructura $y$, educación ambiental tienen el mayor número de relaciones encontradas.

\section{DISCUSIÓN}

Respecto a la calidad educativa, ambas universidades no estarían cumpliendo a cabalidad con los fines y roles para los cuales fueron creados, pues no están proporcionando a la sociedad su estructura social necesaria para controlar el conocimiento más sofisticado disponible, no están contribuyendo significativamente al crecimiento y a la competitividad de la economía regional y nacional; ni a la integración y creación de la sociedad. Tampoco están colaborando con el fortalecimiento y perfeccionamiento de las instituciones necesarias para la gobernabilidad democrática.

Ello se desprende del análisis realizado a los lineamientos "proyecto institucional", "programa académico", "docentes", "proyección social y extensión universitaria" en las que se encontraron discrepancias que fluctúan entre 29 y $50 \%$.

Respecto a la educación ambiental, los resultados obtenidos demuestran que en ambas universidades no existe un compromiso, por parte de sus integrantes, con la educación ambiental ni con la protección al medio ambiente, lo cual explicaría las deficiencias encontradas respecto a conocimientos ambientales de docentes, estudiantes y administrativos (desaprobados en promedio con nota de 8,56 ), no cultivan valores ambientales (con $87 \%$ de discrepancias), ni actitudes ambientales (con $67 \%$ de discrepancias).

Respecto a la relación de la calidad educativa y la educación ambiental, el análisis realizado demuestra que existen 260 relaciones significativas entre calidad educativa y educación ambiental en las universidades estudiadas, lo que confirma la hipótesis de investigación.

De lo que se concluye que las dos variables estudiadas (calidad educativa y educación ambiental) se relacionan 
recíprocamente, lo cual por lo menos permite suponer que existe un factor común que los induce a ello. Podría encontrarse también relaciones de causalidad entre ambas, pero el tratamiento realizado en la investigación no permite por el momento aseverarlo.

Finalmente el reporte de las pruebas de confiabilidad después de la corrida del SPSS, indica que el instrumento de calidad educativa arroja una confiabilidad de 0,70; considerada como buena, al igual que el reporte de la prueba de confiabilidad de educación ambiental que fue de 0,65 , que también puede ser considerado como buena.

\section{REFERENCIAS BIBLIOGRÁFICAS}

Catalán , \& Catany. (1993). La educación ambiental en la enseñanza secundaria. Madrid: Miraquano.

Colom , C. A. (1988). Curriculum y educación ambiental. I Jornada de educación ambiental en la comunidad Valenciana. Valencia.

García, J. (1997). La educación ambiental vertebradora del desarrollo sostenible. Il Congreso Internacional de Universidades para el desarrollo sostenible y el medio ambiente. Granada.

Giménez Martínez, L. (1992). La educación ambiental en la Región de Murcia: situación y perspectivas.

González , E. G. (1995). El desarrollo sustentable. Una alternativa de política institucional. Tialpan: SEMARNAP.
González Faraco, J. C. (1996). Bases socioantropológicas para una intervención educativa en el entorno del Parque Nacional de Doñana.

Junyent, P., \& Geli de Ciuranu , A. (1997). Educación Ambiental: una visión prospectiva de Educación. Ponencias y comunicaciones. Salamanca.

López, G. O. (1966). Educación ambiental y desarrollo humano. Congress International of estrategias and practices in environmental education. Santiago de Compostela.

Oliva, M. (1997). La universidad de la Habana y su papel en la construcción del desarrollo sostenible. I Congreso Internacional de Universidad por el Desarrollo sostenible y el medio ambiente. Granada España.

Pardo, D. A. ( 1995). La educación ambiental como proyecto. Barcelona: Horsori.

Taller de Educación y Capacitación Ambiental (TECA. (1991). Necesidades y fundamentos de la educación ambiental Documento de trabajo № 1 . Santiago: TECA.

UNESCO. (1997). Educación para un Futuro Sostenible: Una visión transdisciplinaria para una acción concertada. Paris.

UNESCO. (1997). Medio ambiente y sociedad: Educación y Sensibilización pública para la sostenibilidad. Conferencia Internacional Thessaloniki. Salónica.

Zarabozo Mila, M. V. (2000). Las Normas Ambientales como Medidas Para-Arancelarias en el Mercado Global. Universidad del País Vasco. 